MMCA-97 Conference, pp. 160-166

R. Čiegis (Ed)

(C) 1997 Vilniaus Gedimino technikos universitetas

\title{
REGULARIZATION BY NONLOCAL CONDITIONS OF THE INCORRECT PROBLEMS FOR DIFFERENTIAL-OPERATOR EQUATIONS OF THE FIRST ORDER
}

\author{
N.I. YURCHUK and ABABNEH MOUSA
}

Belarussian State University

Fr.Skorina Avenue 4,

220050 Minsk, Belarus

\section{SECTION 1}

We consider the differential-operator equation

$$
\frac{d u(t)}{d t}+A u(t)=0
$$

in the interval $(0, T)$, where $u$ is the function of a variable $t \in[0, T]$ with values in the Hilbertian space $H$, and $A$ is a non-bounded self-adjoint operator in $H$.

It is assumed that the operator $A$ does not have a fixed sign and has a bounded reciprocal operator $A^{-1}$ within $H$. Without any loss in generality, for simplicity we assume that the operator $A$ has a point spectrum. An example of this type of operator is the operator in $L_{2}(\Omega)$ generated by the operator $\Delta^{2}$ ( $\Delta$ is the Laplacian given in a bounded dimain $\Omega \subset R^{2}$ ) and boundary conditions at the boundary $\partial \Omega$, giving together with the operator $\Delta^{2}$ a self-adjoint noncoercitive problem (see [1], p.397). The Cauchy problem for the eq. (1) with conditions of the form

$$
u(0)=\chi \quad \text { or } \quad u(T)=\chi
$$

is not correct in sense of Hadamar - Petrovski.

In the present work we have shown that the conditions (2) may be understood as follows. We introduce nonlocal boundary conditions to eq. (1) in the 
following way :

$$
\alpha u(0)+(1-\alpha) u(T)=\chi, \quad \chi \in H, \quad 0<\alpha<1 .
$$

Then, the problem $(1),(3)$ has a strongly generalized solution $U(t, \alpha)$ having the following properties :

$$
\lim _{\alpha \rightarrow 0}\|u(T, \alpha)-\chi\|=0 ; \quad \lim _{\alpha \rightarrow 1}\|u(0, \alpha)-\chi\|=0
$$

where $\|\cdot\|$ is norm in space $H$.

\section{SECTION 2}

Now we demonstrate that the problem (1), (3) has a strongly generalized solution.

Let $\left\{\lambda_{n}\right\}_{n \geq 1}$ be positive eigenvalues of the operator $A$, and $\left\{\mu_{n}\right\}_{n \geq 1}$ be negative eigenvalues of that operator. Let us denote the eigenvectors of the operator $A$ corresponding to the eigenvalues $\left\{\lambda_{n}\right\}_{n \geq 1}$ by $\left\{v_{n}\right\}_{n} \geq 1$ and those corresponding to the eigenvalues $\left\{\mu_{n}\right\}_{n \geq 1}-\left\{w_{n}\right\}_{n \geq 1}$.

Eigenvectors $v_{n}$ and $w_{n}$ of the operator $A$ are forming in $H$ a complete orthogonal system. Without loss in generality, we assume that $\left\|v_{n}\right\|=1$ and ||$w_{n} \|=1$.

Let the vector $\chi \in H$ be represented as

$$
\chi=\sum_{n=1}^{\infty} c_{n} v_{n}+\sum_{n=1}^{\infty} d_{n} w_{n},
$$

where

$$
c_{n}=\left(v_{n}, \chi\right), \quad d_{n}=\left(w_{n}, \chi\right),
$$

and $(.,$.$) is a scolar product in H$.

The solution $u(t, \alpha)$ of eq. (1) is soughtin the form

$$
u(t, \alpha)=\sum_{n=1}^{\infty} \varphi_{n} e^{-\lambda_{n} t} v_{n}+\sum_{n=1}^{\infty} \psi_{n} e^{\mu_{n}(T-t)} w_{n} .
$$

substituting this expression into the conditions of (3) and using the representation of (5), we obtain the following equality :

$$
\sum_{n=1}^{\infty}\left[\alpha+(1-\alpha) e^{-\lambda_{n} T}\right] \varphi_{n} v_{n}+\sum_{n=1}^{\infty}\left[\alpha e^{\mu_{n} T}+(1-\alpha)\right] \psi_{n} w_{n}=
$$




$$
\sum_{n=1}^{\infty} c_{n} v_{n}+\sum_{n=1}^{\infty} d_{n} w_{n}
$$

From this it is inferred that

$$
\begin{gathered}
\varphi_{n}=\left[\alpha+(1-\alpha) e^{-\lambda_{n} T}\right]^{-1} c_{n}, \\
\psi_{n}=\left[\alpha e^{\mu_{n} T}+(1-\alpha)\right]^{-1} d_{n} .
\end{gathered}
$$

Substituting the values $\varphi_{n}$ and $\psi_{n}$ into (7), we have

$$
\begin{aligned}
u(t, \alpha)= & \sum_{n=1}^{\infty} e^{-\lambda_{n} t}\left[\alpha+(1-\alpha) e^{-\lambda_{n} T}\right]^{-1}\left(v_{n}, \chi\right) v_{n} \\
& +\sum_{n=1}^{\infty} e^{\mu_{n}(T-t)}\left[\alpha e^{\mu_{n} T}+(1-\alpha)\right]^{-1}\left(w_{n}, \chi\right) w_{n}
\end{aligned}
$$

Since

$$
\begin{aligned}
\left|e^{-\lambda_{n} t}\left[\alpha+(1-\alpha) e^{-\lambda_{n} T}\right]^{-1}\right| & \leq \frac{1}{\alpha} \\
-\left|e^{\mu_{n}(T-t)}\left[\alpha e^{\mu_{n} T}+(1-\alpha)\right]^{-1}\right| & \leq \frac{1}{1-\alpha},
\end{aligned}
$$

then

$$
\begin{aligned}
& \sup _{0 \leq t \leq T}\left\|\sum_{n=m}^{m+p} e^{-\lambda_{n} t}\left[\alpha+(1-\alpha) e^{-\lambda_{n} T}\right]^{-1}\left(\nu_{n}, \chi\right) \nu_{n}\right\|^{2} \\
& +\sup _{0 \leq t \leq T}\left\|\sum_{n=m}^{m+p} e^{\mu_{n}(T-t)}\left[\alpha e^{\mu_{n} T}+(1-\alpha)\right]^{-1}\left(w_{n}, \chi\right) w_{n}\right\|^{2} \\
& \leq \frac{1}{\alpha^{2}} \sum_{n=m}^{m+p}\left(v_{n}, \chi\right)^{2}+\frac{1}{(1-\alpha)^{2}} \sum_{n=m}^{m+p}\left(w_{n}, \chi\right)^{2}
\end{aligned}
$$

and consequently the series (11) converge to the space norm $C([0, T], H)$ for the functions continuous on $[0, T]$ and possessing the values in $H$.

Thus, for any $\chi \in H$ the problem (1),(3) has a solution strongly generalixed in $C([0, T], H)$, that is representable as a series (11), and for this solution the 
following estimate is correct:

$$
\sup _{0 \leq t \leq T}\|u(t, \alpha)\|^{2} \leq \frac{1}{\alpha^{2}} \sum_{n=1}^{\infty}\left(\nu_{n}, \chi\right)^{2}+\frac{1}{(1-\alpha)^{2}} \sum_{n=1}^{\infty}\left(w_{n}, \chi\right)^{2} .
$$

\section{SECTION 3}

In this paragraph it is demonstrated that the solution $u(t, \alpha)$ posesses the properties of (4). Firstly, it is shown that

$$
\lim _{\alpha \rightarrow 0}\|\chi-u(T, \alpha)\|=0, \quad \forall \chi \in H
$$

Let us consider the difference

$$
\begin{aligned}
\chi-u(T, \alpha)=\quad & \sum_{n=1}^{\infty} \frac{\alpha+(1-\alpha) e^{-\lambda_{n} T}-e^{-\lambda_{n} T}}{\alpha+(1-\alpha) e^{-\lambda_{n} T}}\left(v_{n}, \chi\right) v_{n} \\
& +\sum_{n=1}^{\infty} \frac{\alpha e^{\mu_{n} T}+(1-\alpha)-1}{\alpha e^{\mu_{n} T}+(1-\alpha)}\left(w_{n}, \chi\right) w_{n} .
\end{aligned}
$$

For all $\alpha \in[0,1]$ the value

$$
\frac{\alpha+(1-\alpha) e^{-\lambda_{n} T}-e^{-\lambda_{n} T}}{\alpha+(1-\alpha) e^{-\lambda_{n} T}}=\frac{\alpha\left(1-e^{-\lambda_{n} T}\right)}{\alpha+(1-\alpha) e^{-\lambda_{n} T}}
$$

is nonnegative and nondecreasing with $\alpha$ since its derivative with respect to $\alpha$ is as follows:

$$
\frac{\left(1-e^{-\lambda_{n} T}\right) e^{-\lambda_{n} T}}{\left(\alpha+(1-\alpha) e^{-\lambda_{n} T}\right)^{2}} \geq 0 .
$$

Therefore, the value (17) takes the greatest value at $\alpha=1$, i.e.

$$
0 \leq \frac{\alpha\left(1-e^{-\lambda_{n} T}\right)}{\alpha+(1-\alpha) e^{-\lambda_{n} T}} \leq 1-e^{-\lambda_{n} T} \leq 1 .
$$

For all $\alpha \in[0,1 / 2]$ the value

$$
\frac{\alpha e^{\mu_{n} T}+(1-\alpha)-1}{\alpha e^{\mu_{n} T}+(1-\alpha)}=\frac{\alpha\left(e^{\mu_{n} T}-1\right)}{\alpha e^{\mu_{n} T}+(1-\alpha)}
$$

is nonpositive and nonincreasing with $\alpha$ inasmuch as its derivative with respect to $\alpha$ is as follows :

$$
\frac{\left(e^{\mu_{n} T}-1\right)}{\left(\alpha e^{\mu_{n} T}+(1-\alpha)\right)^{2}} \leq 0 .
$$


Hence the value of (19) on the segment $[0,1 / 2]$ is the lowest when $\alpha=1 / 2$, i.e.

$$
-1 \leq \frac{e^{\mu_{n} T}-1}{e^{\mu_{n} T}+1} \leq \frac{\alpha e^{\mu_{n} T}+(1-\alpha)-1}{\alpha+(1-\alpha) e^{-\lambda_{n} T}} \leq 0 .
$$

Based on the inequalities (18) and (20), from eq. (16) it is inferred that uniformly in $\alpha \in[0,1 / 2]$ the following estimate is true :

$$
\begin{aligned}
\|\chi-u(t, \alpha)\|^{2}= & \sum_{n=1}^{\infty} \frac{\left(\alpha+(1-\alpha) e^{-\lambda_{n} T}-e^{-\lambda_{n} T}\right)^{2}}{\left(\alpha+(1-\alpha) e^{-\lambda_{n} T}\right)^{2}}\left(v_{n}, \chi\right)^{2} \\
& +\sum_{n=1}^{\infty} \frac{\left(\alpha e^{\mu_{n} T}+(1-\alpha)-1\right)^{2}}{\left(\alpha e^{\mu_{n} T}+(1-\alpha)\right)^{2}}\left(w_{n}, \chi\right)^{2} \\
\leq & \sum_{n=1}^{\infty}\left(v_{n}, \chi\right)^{2}+\sum_{n=1}^{\infty}\left(w_{n}, \chi\right)^{2}=\|\chi\|^{2} .
\end{aligned}
$$

Now we demonstrate that

$$
\lim _{\alpha \rightarrow 0}\|\chi-u(T, \alpha)\|^{2}=0, \quad \forall \chi \in M
$$

where $M$ is some set dense in $H$.

Then by virtue of the Banach-Steinhaus theorem, (15) is derived from (21) and (22).

As a set $M$ we take all $\chi$ of the form [see also (5)] :

$$
\chi=\sum_{n=1}^{N} c_{n} v_{n}+\sum_{n=1}^{\infty} d_{n} w_{n}, \quad \forall N<\infty .
$$

Thereupon,

$$
\begin{aligned}
\|\chi-u(t, \alpha)\|^{2}= & \sum_{n=1}^{N} \frac{\alpha^{2}\left(1-e^{-\lambda_{n} T}\right)^{2}}{\left(\alpha+(1-\alpha) e^{-\lambda_{n} T}\right)^{2}}\left(v_{n}, \chi\right)^{2} \\
& \quad+\sum_{n=1}^{\infty} \frac{\alpha^{2}\left(e^{\mu_{n} T}-1\right)^{2}}{\left(\alpha e^{\mu_{n} T}+(1-\alpha)\right)^{2}}\left(w_{n}, \chi\right)^{2} \\
\leq & \frac{\alpha^{2} e^{2 \lambda_{N} T}}{(1-\alpha)^{2}} \sum_{n=1}^{N}\left(v_{n}, \chi\right)^{2}+\frac{\alpha^{2}}{(1-\alpha)^{2}} \sum_{n=1}^{\infty}\left(w_{n}, \chi\right)^{2} \\
= & \frac{\alpha^{2} e^{2 \lambda_{N} T}}{(1-\alpha)^{2}}\|\chi\|^{2}
\end{aligned}
$$

and for such $\chi(22)$ is valid. 
Now we prove that

$$
\lim _{\alpha \rightarrow 1}\|u(0, \alpha)-\chi\|=0, \quad \forall \chi \in H .
$$

Let us consider the difference

$$
\begin{aligned}
\chi-u(0, \alpha)= & \sum_{n=1}^{\infty} \frac{\alpha+(1-\alpha) e^{-\lambda_{n} T}-1}{\alpha+(1-\alpha) e^{-\lambda_{n} T}}\left(v_{n}, \chi\right) v_{n} \\
& +\sum_{n=1}^{\infty} \frac{\alpha e^{\mu_{n} T}+(1-\alpha)-e^{\mu_{n} T}}{\alpha e^{\mu_{n} T}+(1-\alpha)}\left(w_{n}, \chi\right) w_{n}
\end{aligned}
$$

For all $\alpha \in[1 / 2,1]$ the value

$$
\frac{\alpha+(1-\alpha) e^{-\lambda_{n} T}-1}{\alpha+(1-\alpha) e^{-\lambda_{n} T}}=\frac{(\alpha-1)\left(1-e^{-\lambda_{n} T}\right)}{\alpha+(1-\alpha) e^{-\lambda_{n} T}}
$$

is nonnegative and nondecreasing with $\alpha$ since its derivative with respect to $\alpha$ is as follows:

$$
\frac{1-e^{-\lambda_{n} T}}{\left(\alpha+(1-\alpha) e^{-\lambda_{n} T}\right)^{2}} \geq 0
$$

Consequently, the value (25) on the segment $[1 / 2,1]$ takes on the lowest value when $\alpha=1 / 2$, i.e.

$$
-1 \leq \frac{e^{-\lambda_{n} T}-1}{e^{-\lambda_{n} T}+1} \leq \frac{(\alpha-1)\left(1-e^{-\lambda_{n} T}\right)}{\alpha+(1-\alpha) e^{-\lambda_{n} T}} \leq 0
$$

For all $\alpha \in[0,1]$ the value

$$
\frac{\alpha e^{\mu_{n} T}+(1-\alpha)-e^{\mu_{n} T}}{\alpha e^{\mu_{n} T}+(1-\alpha)}=\frac{(1-\alpha)\left(1-e^{\mu_{n} T}\right)}{\alpha e^{\mu_{n} T}+(1-\alpha)}
$$

is nonnegative and nonincreasing with $\alpha$ since its derivative with respect to $\alpha$ is as follows:

$$
\frac{e^{\mu_{n} T}\left(e^{\mu_{n} T}-1\right)}{\left(\alpha e^{\mu_{n} T}+(1-\alpha)\right)^{2}} \leq 0
$$

Therefore, the value (27) is the greatest for $\alpha=0$, i.e.

$$
0 \leq \frac{(1-\alpha)\left(1-e^{\mu_{n} T}\right)}{\alpha e^{\mu_{n} T}+(1-\alpha)} \leq 1-e^{\mu_{n} T} \leq 1
$$


Based on the inequalities (26) and (28), from (24) it is inferred that uniformly in $\alpha \in[1 / 2,1]$ the following estimate is true:

$$
\begin{aligned}
\|\chi-u(0, \alpha)\|^{2}= & \sum_{n=1}^{\infty} \frac{\left(\alpha+(1-\alpha) e^{-\lambda_{n} T}-1\right)^{2}}{\left(\alpha+(1-\alpha) e^{-\lambda_{n} T}\right)^{2}}\left(v_{n}, \chi\right)^{2} \\
& +\sum_{n=1}^{\infty} \frac{\left(\alpha e^{\mu_{n} T}+(1-\alpha)-e^{\mu_{n} T}\right)^{2}}{\left(\alpha e^{\mu_{n} T}+(1-\alpha)\right)^{2}}\left(w_{n}, \chi\right)^{2} \\
\leq & \sum_{n=1}^{\infty}\left(v_{n}, \chi\right)^{2}+\sum_{n=1}^{\infty}\left(w_{n}, \chi\right)^{2}=\|\chi\|^{2} .
\end{aligned}
$$

Now we show that

$$
\lim _{\alpha \rightarrow 1}\|u(0, \alpha)-\chi\|=0, \quad \forall \chi \in M
$$

where $M$ is a particular set dense in $H$. Then according to the BanachSteinhaus theorem, (23) results from (29) and (30). In this case as a set $M$ we take all $\chi$ representable in the form given below:

$$
\chi=\sum_{n=1}^{\infty} c_{n} v_{n}+\sum_{n=1}^{N} d_{n} w_{n}, \quad \forall N<\infty .
$$

Therefore,

$$
\begin{aligned}
\|\chi-u(0, \alpha)\|^{2}= & \sum_{n=1}^{\infty} \frac{(\alpha-1)^{2}\left(1-e^{-\lambda_{n} T}\right)^{2}}{\left(\alpha+(1-\alpha) e^{-\lambda_{n} T}\right)^{2}}\left(v_{n}, \chi\right)^{2} \\
& +\sum_{n=1}^{N} \frac{(1-\alpha)^{2}\left(1-e^{\mu_{n} T}\right)^{2}}{\left(\alpha e^{\mu_{n} T}+(1-\alpha)\right)^{2}}\left(w_{n}, \chi\right)^{2} \\
\leq & \frac{(1-\alpha)^{2}}{\alpha^{2}} \sum_{n=1}^{\infty}\left(v_{n}, \chi\right)^{2}+\frac{(1-\alpha)^{2}}{\alpha^{2}} e^{-2 \mu_{N} T} \sum_{n=1}^{N}\left(w_{n}, \chi\right)^{2} \\
\leq & \frac{(1-\alpha)^{2}}{\alpha^{2}} e^{-2 \mu_{N} T}\|\chi\|^{2}
\end{aligned}
$$

and for such $\chi$ the expressions (30) are true.

\section{REFERENCES}

[1] Brish N.I., Valeshkevith I.N. The Fourier Method for Nonstationary Equations with General Boundary Conditions. Differential Equations 1(3), 1965, P. 393-399. 